\title{
A Lifelogging Platform Towards Detecting Negative Emotions in Everyday Life using Wearable Devices
}

\author{
Chelsea Dobbins \\ Department of Computer Science \\ Liverpool John Moores University \\ Liverpool, UK \\ C.M.Dobbins@ljmu.ac.uk
}

\author{
Stephen Fairclough \\ School of Natural Sciences and \\ Psychology \\ Liverpool John Moores Universit \\ Liverpool, UK \\ S.Fairclough@1jmu.ac.uk
}

\author{
Paulo Lisboa \\ Department of Applied \\ Mathematics \\ Liverpool John Moores University \\ Liverpool, UK \\ P.J.Lisboa@ljmu.ac.uk
}

\author{
Félix Fernando González Navarro \\ Instituto de Ingeniería \\ Universidad Autónoma de Baja \\ California \\ Mexicali, Baja California, Mêxico \\ fernando.gonzalez@uabc.edu.mx
}

\begin{abstract}
Repeated experiences of negative emotions, such as stress, anger or anxiety, can have long-term consequences for health. These episodes of negative emotion can be associated with inflammatory changes in the body, which are clinically relevant for the development of disease in the long-term. However, the development of effective coping strategies can mediate this causal chain. The proliferation of ubiquitous and unobtrusive sensor technology supports an increased awareness of those physiological states associated with negative emotion and supports the development of effective coping strategies. Smartphone and wearable devices utilise multiple on-board sensors that are capable of capturing daily behaviours in a permanent and comprehensive manner, which can be used as the basis for self-reflection and insight. However, there are a number of inherent challenges in this application, including unobtrusive monitoring, data processing, and analysis. This paper posits a mobile lifelogging platform that utilises wearable technology to monitor and classify levels of stress. A pilot study has been undertaken with six participants, who completed up to ten days of data collection. During this time, they wore a wearable device on the wrist during waking hours to collect instances of heart rate (HR) and Galvanic Skin Resistance (GSR). Preliminary data analysis was undertaken using three supervised machine learning algorithms: Linear Discriminant Analysis (LDA), Quadratic Discriminant Analysis (QDA) and Decision Tree (DT). An accuracy of $70 \%$ was achieved using the Decision Tree algorithm.
\end{abstract}

Keywords- Wearable Devices; Pervasive Computing; Lifelogging; Emotion Recognition; Classification

\section{INTRODUCTION}

Negative emotions, such as stress and anxiety, are a common occurrence in daily life and can be seen as a characteristic of a reaction to certain situations or triggers [1]. However, frequent and repeated episodes of stress have been associated with the process of inflammation in the human body [2]. Furthermore, the frequency and magnitude of stress that are experienced by the individual may have a cumulative effect on the cardiovascular health [3]. There is evidence that repeated and sustained activation of the sympathetic nervous system, alongside other physiological changes, such as increased adrenaline, cortisol, and levels of proinflammatory cytokines, is associated with agerelated diseases, such as cardiovascular disease, diabetes, arthritis [2]. Given that between $9 \%-11 \%$ of adults in the UK suffer from cardiovascular disease (CVD) [4], those selfregulatory processes underpinning the recognition/reduction of stress assume increased significance with respect to public health.

It is envisioned that lifelogging technologies can be used as a platform to develop self-awareness in order to support coping strategies that reduce the physiological impact of stress. Lifelogging is a form of pervasive computing and can be described as, "a unified digital record of the totality of an individual's experiences, captured multimodally through digital sensors and stored permanently as a personal multimedia archive" [5]. This paradigm allows individuals to automatically collect vast amounts of personal data over their lifetime. These data can then be used to promote self-reflection, selfexperimentation and to support behaviour change. However, in order to avoid the burden of excessive data entry, it is important for lifelogging platforms to record data automatically and unobtrusively. Modern smartphones and wearable devices contain a number of non-intrusive sensors that enable collection of an enormous amount of personal information. The feasibility of these mobile technologies for lifelogging is supported by its increasing affordability and pervasiveness, i.e. sensors are becoming cheaper and smaller. Additionally, smartphones sit within a growing category of networked devices that can be used to monitor the behaviour of the individual [6].

This paper presents a mobile lifelogging platform that has been developed to: (1) collect instances of data, including heart rate (HR) and Galvanic Skin Resistance (GSR) from a wearable device, (2) synchronise and remove noise and artefacts from the collected data, and (3) apply machine learning techniques to classifying instances of stress. Physiological markers of stress are monitored through the collection of psychophysiological data (HR and GSR). The usage of implicit monitoring delivers quantitative data during everyday life, which can be transformed into visualisations that support accurate recall from long-term memory.

The reminder of this paper is constructed as follows. Section 2 provides an overview of background literature, whilst section 3 illustrates the design of the posited system. Section 4 describes the pilot study that was undertaken to collect data in everyday life from undergraduate university students using wearable devices. This section also presents subsequent signal preprocessing and labelling that was undertaken on this data. Section 5 presents results of preliminary analysis to classify stress using the data described in section 4, whilst section 6 


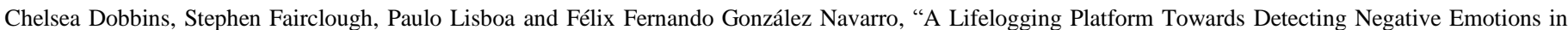

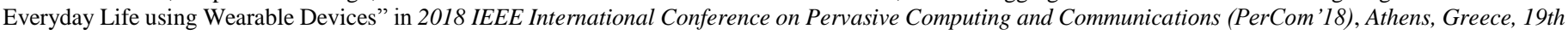
- 23rd March, 2018

presents a discussion of the results. The paper is then concluded in section 7.

\section{BACKGROUND}

Recent advances in wearable sensor technology have provided more efficient means of capturing data and recording information in a digital form.

\section{A. Lifelogging and Sensing Emotion}

Humans have been documenting their personal lives for centuries via the use of diaries and personal journals. Originally, in handwritten form, this outlet allows the person to record a chronological sequence of events, as well as thoughts and feelings recorded at those times [7]. However, this retrospective method suffers from the inherent biases and limitation of reflection and remembrance. The idea of capturing every moment of our lives digitally, saving every piece of information and thus creating archives of personal digital information has existed since the mid 1940's [8].

The advancement of wearable devices are now seen as the ideal platform upon which to collect personal data [9]. Using such devices for lifelogging mitigates issues of keeping handwritten diaries as data is captured 'in the moment'. For instance, the Affective Diary [10] used a range of bio-sensors to record "affective body memorabilia," such as pulse, skin conductivity and activity, and a mobile phone to record SMSs sent/received, photographs taken and the Bluetooth presence of other mobile phones in the vicinity [10]. Similarly, the AffectAura [11], system collected electrodermal activity (EDA) data from a wearable wrist sensor [12], as well as audio, visual, and contextual data. The data was subsequently used to predict valence, arousal and engagement, which was correlated with events on a timeline. The system used a nearest-neighbor classifier, which achieved an overall accuracy of $68 \%$ [11]. In other works, Ayzenberg et al.'s [13] FEEL system used a commercial Affectiva $\mathrm{Q}^{\mathrm{TM}}$ wrist-worn sensor to monitor EDA, skin temperature and acceleration. The system then ereated a "journal of affectively labelled eyents". Additionally, Sano et al. [14] examined academic performance, sleep quality and stress levels. In their work, they also utilized the Affectiva $\mathrm{Q}^{\mathrm{TM}}$ wristworn sensor to measure acceleration, skin temperature and skin conductance and obtained classification accuracies of between $67-92 \%$.

The increased functionality, multimodal capacity and multiple sensors possessed by smartphones and smartwatches make them ideal candidates for self-tracking and quantification of the self [15]. For instance, monitoring negative emotions in everyday life could serve as a pre-emptive system that supports the development of adaptive coping strategies.

\section{B. The Effects of Stress on the Body}

When a person enters a stressful state, they encounter what is commonly known as the "fight or flight" response, a physiological reaction that results from a perceived threat [16]. Acute stress is responsible for diminishing the brains ability to accurately recall memories, however this function is reversible and not permanent [17]. On the other hand, chronic stress can result in the acceleration of biological markers of aging [18]. It has been suggested the level of stress that occurs at an early age can increase or decrease the rate of biological aging [19].

\section{SYSTEM DESIGN}

The availability of wearable sensing devices presents us with unique opportunities to design intelligent systems that can be taken into the real-world in order to monitor the emotional state of users. This requires systems to unobtrusively collect large quantities of data over prolonged periods and process this data to derive meaning. As such, the system posited in Fig. 1 has been designed to: (1) monitor the stress of users through the utilization of wearable technology, and (2) provide feedback to promote self-awareness to develop effective coping strategies. This will be undertaken by focusing on:

1. Collecting instances of lifelogging data

2. Pre-processing and labelling this data

3. Classifying negative emotional states

4. Providing feedback to the user.

The system takes a multimodal approach; whereby multiple streams of data are incorporated into the system before a decision is made and feedback presented. The intention is to collect data from users in their natural environment, over a prolonged period. The wearable sensors used gather physiological markers of stress through the collection of psychophysiological data, including heart rate and skin conductance. Thus, the approach produces results that are quantifiable and objective. A pilot study has been undertaken to explore the feasibility of the system. It should be noted that, the visualization element is out of the scope of this paper and will be the subject of future work.

\section{PILOT StUdY}

Our lifelogging platform has been developed to measure negative emotional states during everyday life. The platform utilizes a commercially available wearable device (Microsoft Band 2) to collect physiological lifelogging data, including heart rate (HR) and Galvanic Skin Resistance (GSR). Data are collected on the Band 2, which then connects to a smartphone, via a purpose-built Android application and Bluetooth, where the data is stored. Fig. 2 depicts the sensor hardware that has been used for the study.

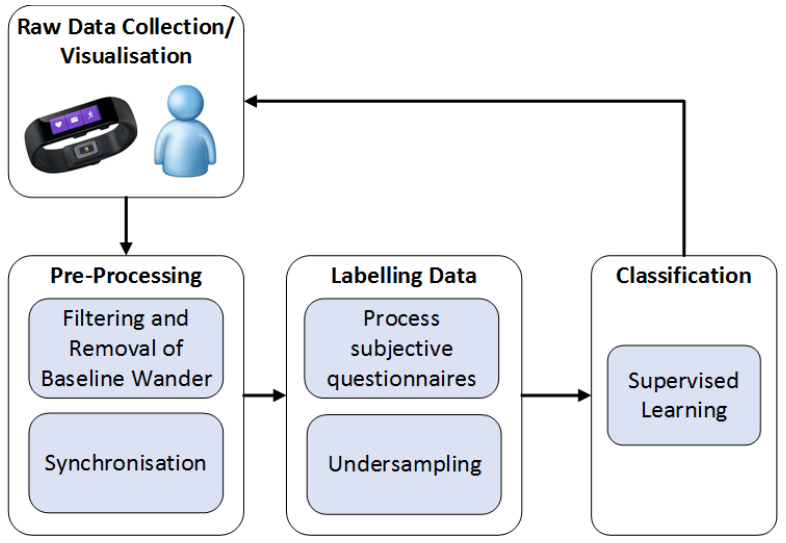

Fig. 1. Design of the mobile lifelogging system 


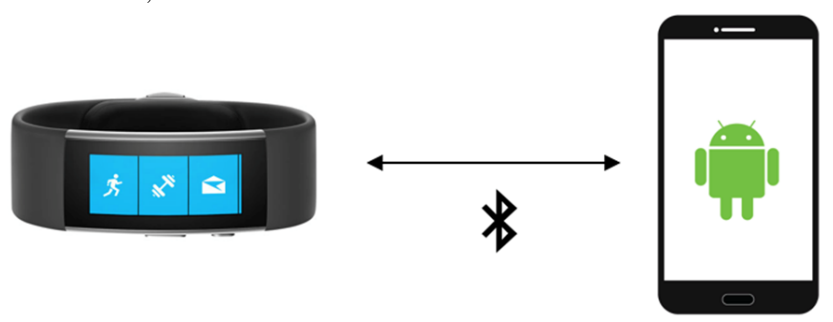

Fig. 2. Mobile sensor equipment used for the pilot study, including Microsoft Band 2 and smartphone

\section{A. Participants and Data Collection}

In order to assess the validity of our approach, the system has been implemented via a pilot study of six undergraduate university students. As an incentive, after completing the study, participants were paid using a gift voucher to the value of $£ 20$.

Physiological lifelogging data, including heart rate (HR) and Galvanic Skin Resistance (GSR), were collected using the Microsoft Band 2. Additionally, the Perceived Stress Scale (PSS) [20] questionnaire was used to collect subjective reports about participants daily levels of stress. Each question was rated on a 5-point Likert scale ranging from never (0) to very often (4). Positively worded items were reverse scored, and the ratings are summed, with higher scores indicating more perceived stress. The responses in this questionnaire are intended to identify how unpredictable, uncontrollable, and overloaded participants find their lives [20]. Participants were provided with the Microsoft Band 2 and were given a demonstration and instructions about how to operate this device. The custom-built Android application was also installed on their phones, which is required to 1) connect to the Band to store data and 2) to administer the PSS questionnaire. The PSS questionnaires were completed twice per day, once in the morning and again in the afternoon and the Band was worn for up to 10 working days, during waking hours. The University Ethical Committee approved all procedures for participant recruitment and data collection prior to commencement of the study. In total, 974,564 instances of data were collected over the course of the study.

\section{B. Signal Pre-Processing}

The pre-processing of the data has been undertaken using MATLAB r2017b. One of the other challenges involved in the collection of large datasets 'in the wild' is the occurrence of artefacts, which can be have a significant influence on the data. Thus, it is an essential requirement for appropriate signal processing methods to be utilized for signal smoothing and baseline drift adjustment. Furthermore, since we are recording data from the wrist over a prolonged period and in the natural environment, as opposed to a lab setting, instances of noise, artefacts and missing data values are likely to appear in our datasets. For instance, loss of contact between the sensor and the skin is inevitable.

Baseline drift is a type of unique artefact that commonly occurs when collecting physiological datasets in the field over long periods of time. These artefacts are typically the result of motion artefacts from the electrode-skin interface, respiration and motion of the subject, which consequentially leads to signal quality slowly degrading over a period of time. Moreover, the selected method must avoid distorting the original signal, as it is important to ensure that the integrity of any classification built using this data is intact. Our approach uses a three-step strategy that first applies an Empirical Mode Decomposition, which extracts a series of Intrinsic Mode Functions (IMF) [21]. These signals contain baseline wander components, as well as the main signal components. Secondly, a morphological filter has been applied in order to extract baseline wander from the IMF [21], [22]. The process of applying a morphological filter involves the modification of an objects shape, by performing a transformation using the output of its interaction with a structuring element. There are four fundamental operators (erosion, dilation, opening and closing) that are typically included within a morphological transformation [23]. We utilise a non-linear top-hat transform approach for removing the baseline drift from the GSR data (see Fig. 3). An integral component of this approach exists in the configuration of what is referred to as the structuring element, this is the reference point that the original signal uses to remove the drifting elements. The baseline wander signals, $b w$, were then combined and subtracted from the original signal, $x(1)$. As Fig. 3 demonstrates, baseline wander has been removed without affecting the integrity of the signal

$$
f=x-\left(\sum b w\right)
$$

The final stage of pre-processing involved synchronizing the signals. Heart rate data from the Microsoft Band 2 was sampled at $1 \mathrm{~Hz}$, whilst GSR was sampled at $5 \mathrm{~Hz}$. Therefore, it was necessary to downsample and lower the sampling rate of the GSR signal in order to synchronize these data with the heart rate signal. This downsampling procedure was achieved by averaging the GSR signal over 5 second epochs. Instances where loss of contact between the Band 2 and the skin occurred were then filtered. The Microsoft Band 2 software development kit (SDK) provides a Boolean check of the state of contact with the skin, whereby $0=$ loss of contact and $1=$ contact is present. All instances where contact $=0$ have been removed.

\section{Data Labelling}

Labels for the data were derived using the PSS subjective questionnaire data. As participants completed the questionnaire twice a day, separate scores for the morning and afternoon were calculated. A change score was then calculated (afternoon morning) to index mood changes that have occurred during the day. Scores can be categorized into two states - A positive increase indicates stress, whilst a negative score indicates stress hasn't occurred.

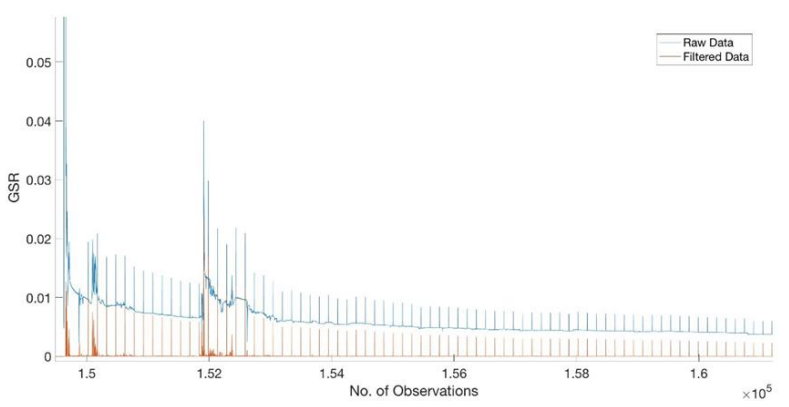

Fig. 3. Raw GSR signal with baseline drift. Blue: before application of morphological filter. Orange: after application of morphological filter 


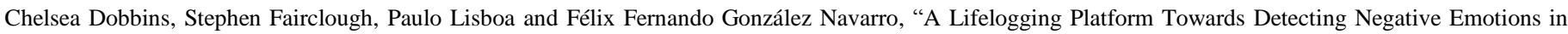

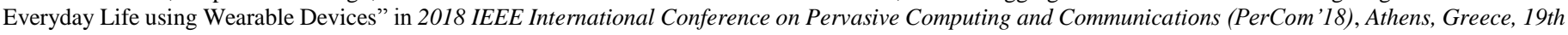
- 23rd March, 2018

A zero indicates that a change hasn't occurred over the course of the day. The data was labelled using the derived states identified in Fig. 4. Data that was collected on days that had a change score of zero (i.e. no change occurred). A consequence of labelling via subjective labels is that the datasets are unbalanced. This can produce bias during the classification process, as a poorer predictive accuracy can occur over the minority class (in this instance the stress class) [24]. Therefore, to correct this issue, the data was randomly undersampled using the SpreadSubsample class in Weka 3.8. This filter produces a random subsample of the dataset, where the maximum count for each class is set to the size of the minority class. This process resulted in $3.71 \%$ of the labelled data being removed. The data for each participant was then combined and normalized into one dataset, which was then used within the preliminary data analysis.

\section{PREliminary Data ANALYsis}

The purpose of this analysis was to classify real-world data to detect instances of stress. This was undertaken using three supervised machine learning algorithms: Linear Discriminant Analysis (LDA), Quadratic Discriminant Analysis (QDA) and Decision Tree (DT). The analysis was performed using the $\mathrm{mlr}$ (Machine Learning in R) package in RStudio v.1.1.383 The classification models were validated using $k$-fold crossvalidation, whereby $k=10$. The performance of each classification model was evaluated using the resulting confusion matrix. An example of a confusion matrix output can be seen in Fig. 5 [25]. Performance measures were then generated from the confusion matrix outputs including:

- False Negative Rate (FNR) - misses that occurs when stressful day has been classified as relaxed:

$$
f n r=\frac{F N}{(F N+T P)}
$$

- False Positive Rate (FPR) - false alarms whereby a relaxed day has been incorrectly classified as being stressful:

$$
f p r=\frac{F P}{(T N+F P)}
$$

- True Positive Rate (TPR) [Recall/Sensitivity] - the number of correctly classified stressful days:

$$
t p r=\frac{T P}{(F N+T P)}
$$

- Positive Predictive Value (PPV) [Precision] - the number of results that have been marked as stressful when stress actually occurred:

$$
p p v=\frac{T P}{(F P+T P)}
$$

- Accuracy - an index of overall performance:

$$
a c c=\frac{(T N+T P)}{(T N+F P+F N+T P)}
$$

- Balanced Error Rate (BER) - the average misclassification error rates of each class:

$$
\text { ber }=0.5 \cdot\left(\frac{F P}{(T N+F P)}+\frac{F N}{(F N+T P)}\right)
$$

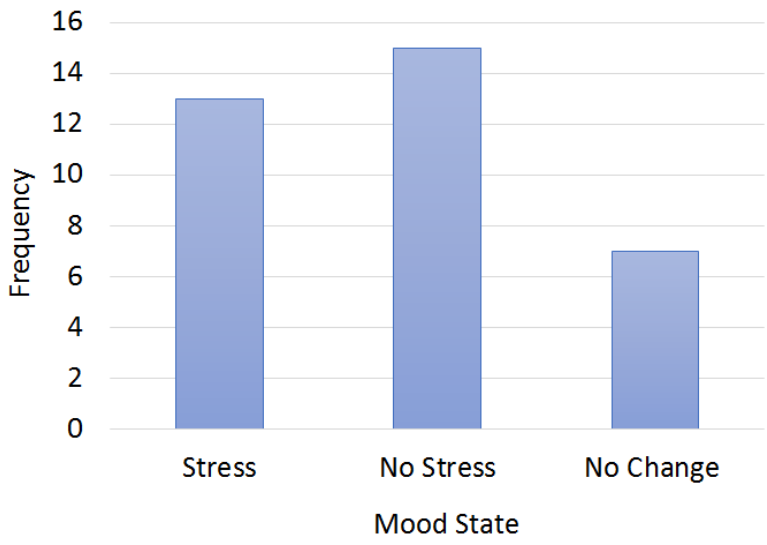

Fig. 4. Self-reported mood that has been identified via the PSS questionnaire

\begin{tabular}{|c|c|c|c|}
\hline \multicolumn{2}{|c|}{} & \multicolumn{2}{c|}{ Predicted Class } \\
\cline { 3 - 4 } & \multicolumn{2}{|c|}{ Negative (Relaxed) } & Positive (Stress) \\
\hline \multirow{2}{*|2}{} & $\begin{array}{c}\text { Negative } \\
\text { (Relaxed) }\end{array}$ & $\begin{array}{c}\text { True Negative (TN) } \\
\text { Correct rejection of } \\
\text { relaxed instances }\end{array}$ & $\begin{array}{c}\text { False Positive (FP) } \\
\text { False alarms }\end{array}$ \\
\cline { 2 - 4 } & $\begin{array}{c}\text { Positive } \\
\text { (Stress) }\end{array}$ & $\begin{array}{c}\text { False Negative (FN) } \\
\text { Misses }\end{array}$ & $\begin{array}{c}\text { True Positive (TP) } \\
\text { Correct identification } \\
\text { of stress }\end{array}$ \\
\hline
\end{tabular}

Fig. 5. Confusion matrix outputs

$F_{1}$ Score - a measurement of the balance between PPV (precision) and TPR (recall/sensitivity):

$$
f=2 \cdot \frac{(P P V \cdot T P R)}{(P P V+T P R)}
$$

Three types of analysis have been undertaken using 1) only the heart rate data, 2) only the GSR data and 3) using both heart rate and GSR data. Fig. 6 illustrates the accuracy results. As it can be seen, independently using heart rate (with all classifiers) and GSR (with LDA and QDA) produced poor results. However, using the decision tree classifier with either the GSR data or with both HR and GSR data produced significantly better results. The BER and $F_{1}$ results (see Fig. 7) illustrates that the lowest error rate was produced using the decision tree classifier with either just the GSR data or with both HR and GSR data.

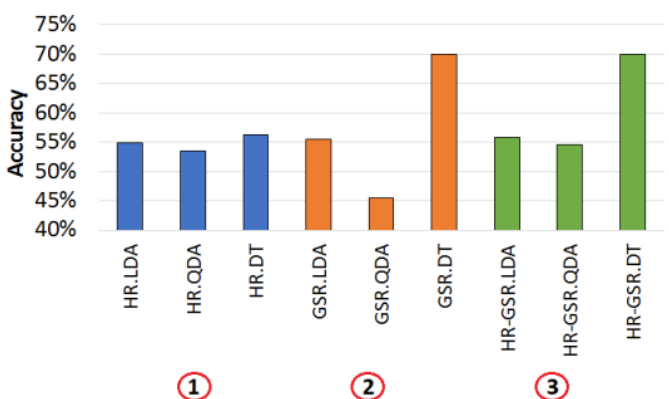

Dataset/Classifier

Fig. 6. Accuracy results for classifying data related to 1) only the heart rate, 2) only GSR and 3) both heart rate and GSR data using Linear Discriminant Analysis (LDA), Quadratic Discriminant Analysis (QDA) and Decision Tree (DT) classifiers 


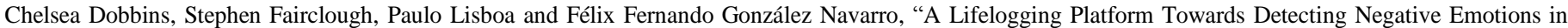

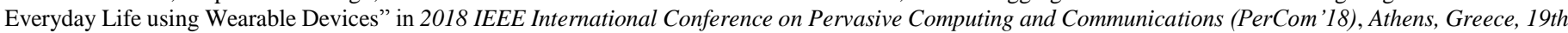
- 23rd March, 2018

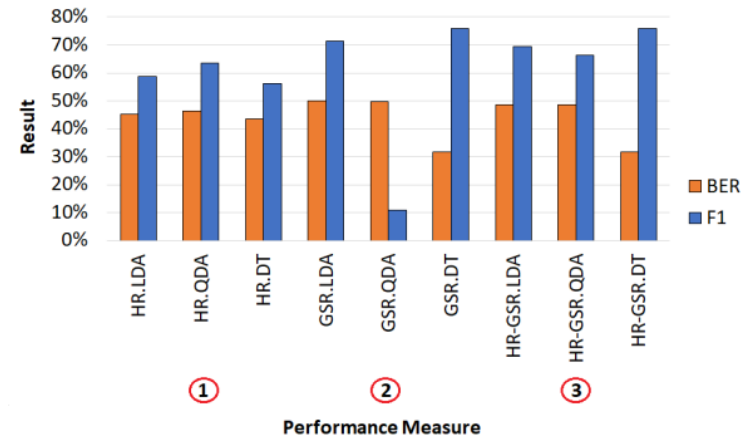

Fig. 7. Balanced Error Rate (BER)and $F_{1}$ results for classifying data related to 1) only the heart rate, 2) only GSR and 3) both heart rate and GSR data using Linear Discriminant Analysis (LDA), Quadratic Discriminant Analysis (QDA) and Decision Tree (DT) classifiers

TABLE I presents the classifier performance results, which demonstrated that the LDA with either the GSR data or with both $\mathrm{HR}$ and GSR data produced missed the least number of stressful days (low FNR) and had the highest sensitivity (TPR). However, QDA with only GSR data produced the lowest false alarm rate (FPR), whilst decision tree with either the GSR data or with both HR and GSR data produced the highest precision (PPV). The performance of each classifier has also been evaluated using a Receiver Operating Characteristic (ROC) plot (see Fig. 8), which plots FPR (false alarms) against TPR (recall/sensitivity). As depicted in Fig. 8, the decision tree classifier with both the GSR data or with both HR and GSR data performed particularly well.

\section{DISCUSSION}

This paper has demonstrated positive results for detecting stress in everyday life. A pilot study has been undertaken to collect physiological data, including heart rate (HR) and Galvanic Skin Resistance (GSR), via the wearable Microsoft Band 2 for up to ten working days in the real-world. The highest accuracy of $70 \%$ was achieved using the decision tree algorithm with GSR data and with both HR and GSR data. It is interesting that the addition of HR did notimprove the classification results.

TABLE I CLASSIFIER PERFormance

\begin{tabular}{|c|c|c|c|c|}
\hline \multirow{2}{*}{ Dataset } & Misses & $\begin{array}{c}\text { False } \\
\text { Alarm }\end{array}$ & Precision & Sensitivity \\
\cline { 2 - 5 } & FNR & FPR & PPV & TPR \\
\hline HR.LDA & $35 \%$ & $55 \%$ & $54 \%$ & $65 \%$ \\
\hline HR.QDA & $20 \%$ & $73 \%$ & $52 \%$ & $80 \%$ \\
\hline HR.DT & $44 \%$ & $43 \%$ & $56 \%$ & $56 \%$ \\
\hline GSR.LDA & $\mathbf{0 \%}$ & $100 \%$ & $55 \%$ & $\mathbf{1 0 0 \%}$ \\
\hline GSR.QDA & $94 \%$ & $\mathbf{5 \%}$ & $59 \%$ & $6 \%$ \\
\hline GSR.DT & $16 \%$ & $48 \%$ & $\mathbf{6 9 \%}$ & $84 \%$ \\
\hline HR-GSR.LDA & $\mathbf{9 \%}$ & $88 \%$ & $56 \%$ & $\mathbf{9 1 \%}$ \\
\hline HR-GSR.QDA & $19 \%$ & $78 \%$ & $56 \%$ & $81 \%$ \\
\hline HR-GSR.DT & $15 \%$ & $48 \%$ & $\mathbf{6 9 \%}$ & $85 \%$ \\
\hline
\end{tabular}

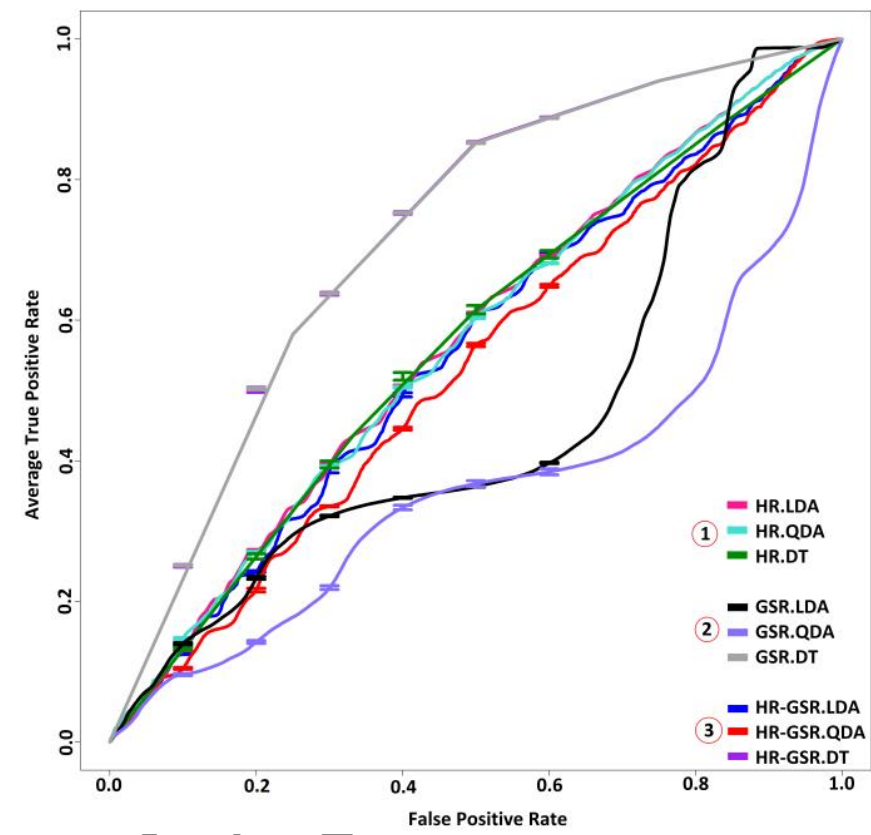

Fig. 8. ROC plot for classifying data related to 1) only the heart rate, 2) only GSR and 3) both heart rate and GSR data using Linear Discriminant Analysis (LDA), Quadratic Discriminant Analysis (QDA) and Decision Tree (DT) classifiers

Nevertheless, the results are encouraging and provide a solid basis to explore and improve upon. If we look ahead to feedback systems, there is inevitably a trade-off between false alarms and misses. If the purpose of a system is monitor a user in everyday life, then a higher rate of false alarms could induce undue stress. However, for clinical purposes whereby negative emotions are is linked to negative health outcomes, a higher rate of false alarms would be more acceptable, as these instances could be investigated further.

A limitation of this work is that it does not consider sleep. Stress is not only experienced during the day but also at night, whilst we sleep [26]. Recording data 24 hours a day would be ideal to examine the manifestation and difference between conscious and unconscious stress. However, the battery of powered devices and contact with water (i.e. when showering or swimming) are issues. Future work will also consider the development of visualizations to provide feedback. This must be carefully considered and be meaningful so as to not cause undue stress but also facilitate self-reflection.

\section{CONCLUSION}

The widespread proliferation of wearable devices offers an alternative means of measuring emotional states ubiquitously and unobtrusively [27]. Wearable devices possess a number of sensors that can collect a variety of data, which can be amalgamated to detect and infer transitory moments of stress. This is important to identify moments of negative emotions that we might be unaware of or to identify the frequency of these episodes.

This work has demonstrated that data collected from wearable devices in everyday life can be used to successfully detect instances of stress. A respectable accuracy of $70 \%$ has 
Chelsea Dobbins, Stephen Fairclough, Paulo Lisboa and Félix Fernando González Navarro, "A Lifelogging Platform Towards Detecting Negative Emotions in Everyday Life using Wearable Devices” in 2018 IEEE International Conference on Pervasive Computing and Communications (PerCom'18), Athens, Greece, 19th - 23rd March, 2018

been achieved using the Decision Tree classification algorithm. The next steps for this work is to explore the integration of feedback. This would enable us to become more aware of our emotional wellbeing and to learn self-regulating negative emotions, which is imperative for leading a healthy lifestyle.

\section{ACKNOWLEDGMENTS}

The authors would like to thank all of the participants for agreeing to take part in this study.

\section{REFERENCES}

[1] R. C. Thurston, M. Rewak, and L. D. Kubzansky, “An Anxious Heart: Anxiety and the Onset of Cardiovascular Diseases," Prog. Cardiovasc. Dis., vol. 55, no. 6, pp. 524-537, Jan. 2013.

[2] B. L. Ganzel, P. A. Morris, and E. Wethington, "Allostasis and the human brain: Integrating models of stress from the social and life sciences.," Psychol. Rev., vol. 117, no. 1, pp. 134-174, Jan. 2010.

[3] M. A. Samuels, "The Brain-Heart Connection," Circulation, vol. 116, no. 1, pp. 77-84, Jul. 2007.

[4] V. Becerra et al., "Cost-effectiveness and public health benefit of secondary cardiovascular disease prevention from improved adherence using a polypill in the UK," BMJ Open, vol. 5, no. 5, pp. e007111-e007111, 2015.

[5] M. Dodge and R. Kitchin, "'Outlines of a world coming into existence': Pervasive computing and the ethics of forgetting," Environ. Plan. B Urban Anal. City Sci., vol. 34, no. 3, pp. 431-445, 2007.

[6] C. Dobbins, R. Rawassizadeh, and E. Momeni, "Detecting Physical Activity within Lifelogs towards Preventing Obesity and Aiding Ambient Assisted Living," Neurocomputing, vol. 230, pp. 110-132, 2017.

[7] J. Machajdik et al., "Providing feedback on emotional experiences and decision making," in IEEE Africon '11, 2011, pp. 1-6.

[8] V. Bush, "As We May Think," The Atlantic Monthly, no. JULY 1945, 1945.

[9] R. Rawassizadeh, E. Momeni, C. Dobbins, P. Mirza-Babaei, and R Rahnamoun, "Lesson Learned from Collecting Quantified Self Information via Mobile and Wearable Devices," Y. Sens. Actuator Networks, vol. 4, no. 4, pp. 315-335, Nov. 2015.

[10] A. Ståhl, K. Höök, M. Svensson, A. S. Taylor, and M. Combetto, "Experiencing the Affective Diary," Pers. Ubiquitous Comput., vol. 13, no. 5, pp. 365-378, Jun. 2009.

[11] D. McDuff, A. Karlson, A. Kapoor, A. Roseway, and M. Czerwinski, "AffectAura: An Intelligent System for Emotional Memory," in Proceedings of the 2012 ACM annual conference on Human Factors in Computing Systems - CHI'12,2012, pp. 849-858.

[12] M. Z. Poh, N. C. Swenson, and R. W. Picard, "A Wearable Sensor for Unobtrusive, Long-Term Assessment of Electrodermal Activity," IEEE Trans. Biomed. Eng., vol. 57, no. 5, pp. 1243-1252, May 2010.

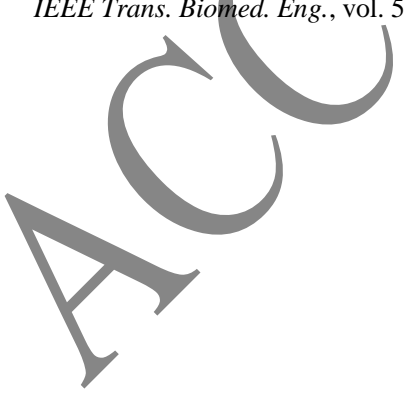

[13] Y. Ayzenberg, J. Hernandez, and R. W. Picard, "FEEL: frequent EDA and event logging -- a mobile social interaction stress monitoring system," in Proceedings of CHI'12, 2012, pp. 2357-2362.

[14] A. Sano et al., "Recognizing academic performance, sleep quality, stress level, and mental health using personality traits, wearable sensors and mobile phones," in 2015 IEEE 12th International Conference on Wearable and Implantable Body Sensor Networks, BSN 2015, 2015, pp. 1-6.

[15] T. Minh, T. Do, J. Blom, and D. Gatica-perez, "Smartphone Usage in the Wild: a Large-Scale Analysis of Applications and Context," ICMI '11 Proc. 13th Int. Conf. multimodal interfaces, pp. 353-360, 2011.

[16] P. Paredes, R. Giald-Bachrach, M. Czerwinski, A. Roseway, K. Rowan, and J. Hernandez, "PopTherapy: Coping with Stress through Pop-Culture," in Proceedings of the 8th International Conference on Pervasive Computing Technologies for Healthcare (PervasiveHealth'14), 2014, pp. 109-117.

[17] C. Kirschbaum, O. T. Wolf, M. May, W. Wippich, and D. H. Hellhammer, "Stress- and treatment-induced elevations of cortisol levels associated with impaired declarative memory in healthy adults," Life Sci., vol 58, no. 17, pp. 1475-1483, Mar. 1996.

[18] D. S. Kerr, L. W. Campbell, M. D. Applegate, A. Brodish, and P. W. Landfield, "Chronic stress-induced acceleration of electrophysiologic and morphometric biomarkers of hippocampal aging," J. Neurosci., vol. 11, no. 5, pp 1316-1324, May 1991.

[19] B. S. McEwen, "Stress, Adaptation, and Disease: Allostasis and Allostatic Load," Ann. N. Y. Acad. Sci., vol. 840, no. 1, pp. 33-44, May 1998.

[20] S. Cohen, "Perceived Stress Scale," Psychology, pp. 1-3, 1994.

[21] T. Y. Ji, Z. Lu, Q. H. Wu, and Z. Ji, "Baseline normalisation of ECG signals using empirical mode decomposition and mathematical morphology," Electron. Lett., vol. 44, no. 2, p. 82, 2008.

[22] A. C. Sauve and T. P. Speed, "Normalization, Baseline Correction and Alignment of High-Throughput Mass Spectrometry Data." M. Sedaaghi and Q. Wu, "Real-time implementation of grey-scale morphological operators," vol. 33, no. 21, pp. 1761-1763, 1997. N. V. Chawla, A. Lazarevic, L. O. Hall, and K. Bowyer, "SMOTEBoost: Improving Prediction of the Minority Class in Boosting," Proc. Princ. Knowl. Discov. Databases, PKDD-2003, pp. 107-119, 2003.

[25] R. Kohavi and F. Provost, "Glossary of Terms," Mach. Learn., vol. 30, no. 2/3, pp. 271-274, 1998.

[26] A. Muaremi, A. Bexheti, F. Gravenhorst, B. Arnrich, and G. Troster, "Monitoring the Impact of Stress on the Sleep Patterns of Pilgrims using Wearable Sensors," in IEEE-EMBS International Conference on Biomedical and Health Informatics (BHI), 2014, pp. 185-188.

[27] S. Greene, H. Thapliyal, and A. Caban-Holt, "A Survey of Affective Computing for Stress Detection: Evaluating technologies in stress detection for better health.," IEEE Consumer Electronics Magazine, vol. 5, no. 4. pp. 44-56, Oct-2016. 